\title{
Functional Models for Congenital Anomalies of the Kidney and Urinary Tract
}

\author{
Glenn van de Hoek ${ }^{a}$ b Nayia Nicolaou ${ }^{a}$ Rachel H. Giles ${ }^{b}$ Nine V.A.M. Knoers ${ }^{a}$ \\ Kirsten Y. Renkema ${ }^{a}$ Ernie M.H.F. Bongers ${ }^{c}$ \\ ${ }^{a}$ Department of Medical Genetics, Center for Molecular Medicine, ${ }^{b}$ Department of Nephrology and Hypertension, \\ University Medical Center Utrecht, Utrecht, and ' Department of Human Genetics, Radboud University Medical Center, \\ Nijmegen, The Netherlands
}

\begin{abstract}
Key Words
CAKUT · Functional characterization · Gene · Kidney ·

Model system · Mutation
\end{abstract}

\begin{abstract}
Congenital anomalies of the kidney and urinary tract (CAKUT) constitute one of the most common developmental diseases in humans; however, the cause for most patients remains unknown. Efforts to identify novel genetic causes for CAKUT through next-generation sequencing techniques have led to the discovery of new genes and risk factors. Concomitantly, these same efforts have generated large gene candidate lists requiring individual functional characterization. Appropriate model systems are needed to assess the functionality of genes and pathogenicity of genetic variants discovered in CAKUT patients. In this review, we discuss how cellular, animal, and personal (human) models are being used to study CAKUT candidate genes and what their major advantages and disadvantages are with respect to relevance and throughput.
\end{abstract}

(c) 2014 S. Karger AG, Basel

\section{Introduction}

The development of the renal system entails a complex process of interactions between tissues, genetic pathways, and environmental factors, which, when disturbed, can lead to congenital anomalies of the kidney and urinary tract (CAKUT). The molecular mechanisms underlying human embryonic kidney and urinary tract development, including the processes that may lead to malformation, have been extensively reviewed [1]. In brief, human kidney development proceeds through three stages: the pronephros, mesonephros, and metanephros. Both the pronephros and mesonephros are transient structures and although they do not contribute structurally to the eventual metanephros, they do provide inductive signals for adult kidney development [2]. Coordinated interactions between the early nephrotic tissues are fundamental to ureteric budding, epithelial branching, mesenchymal to epithelial transformation, morphogenesis, and differentiation. Although these processes are crucial for normal development of the kidneys and urinary tract, malfunction of the same processes were shown to be involved in CAKUT [3]. The advent of next-generation sequencing (NGS) techniques has enabled extensive sequencing efforts in patients with renal disease to identify novel underlying molecular mechanisms [4] and has led to a plethora of prospective CAKUT candidate genes. Proving causality of the genetic variants identified, is one of the challenges in CAKUT research. Here, we summarize the models that are currently available and discuss what is

Glenn van de Hoek and Nayia Nicolaou contributed equally to this work.

\section{KARGER}

E-Mail karger@karger.com

www.karger.com/nef
(C) 2014 S. Karger AG, Basel

$1660-8151 / 14 / 1291-0062 \$ 39.50 / 0$
Dr. Kirsten Y. Renkema

Department of Medical Genetics, STR.1.305, University Medical Center Utrecht PO Box 85060

NL-3508 AB Utrecht (The Netherlands)

E-Mail K.Renkema@umcutrecht.nl 
needed to improve (high-throughput) functional characterization of these genes and how this could benefit CAKUT diagnostics and personalized care.

\section{Current Models for CAKUT}

\section{In Vitro Proteomic Analysis}

Proving the pathogenicity of genetic variants identified in patients with CAKUT requires model systems that have an appropriate read-out and fit the organ system and the genes/proteins of interest. Kidney-derived cells, including mpkCCD, HEK293, MDCK, and IMCD3 are among the most widely used cell lines in kidney research and are suitable for the study of gene expression levels, protein localization, and protein-protein interactions. Furthermore, in vitro approaches delivered valuable insights into the pathways governing renal development, for example, the GDNF-RET pathway [5]. Many of these models are readily available and inexpensive, allowing for high-throughput analysis (table 1). Stable cell culture systems are effective for studying biochemical pathways and functions of a gene on the single-cell level. However, they lack structural read-outs on interaction with different cell types or on the whole-organ level, which is an integral aspect of the defects resulting in CAKUT.

\section{In Vivo Models for CAKUT}

Thus far, mice have been favorite models for investigating the role of human mutations in kidney disease, as the development of the mouse metanephros is similar to human. The ability to analyze static serial time points and perform lineage tracing additionally makes mice suitable for developmental research. Mutant mice showing defects in kidney development have been extensively studied and have consequently proposed numerous candidate genes for human CAKUT; Islet1 (Isl1) deletions were shown to cause CAKUT phenotypes in mice, calling for further investigation of potential ISLET1 mutations in CAKUT patients [6]. Moreover, recessive mutations where found in integrin alpha 8 (ITGA8) in patients with bilateral renal agenesis [7], while Itga8 knockout mice were already shown to have aberrant kidney development [8], strongly suggesting a causal role for ITGA 8 mutations in CAKUT. Current developments in 'genome editing' including targeted mutagenesis through zinc finger nucleases, transcription activator-like effector nucleases (TALEN) and the clustered regularly interspaced short palindromic repeats (CRISPR)/Cas9 system, significantly impact the modeling of human genetics into mouse in vivo models [9]. Generating mouse models is, however, time-consuming and expensive and therefore, less suitable for high-throughput efforts (table 1).

The mammalian metanephric mesenchyme can be cultured so that the developmental program of early nephrogenesis can be recapitulated under in vitro/ex vivo conditions (fig. 1a). Culturing murine kidneys ex vivo is a promising tool for research on the developmental aspects of CAKUT [10]. The explants allow for a direct access to kidney tissue and are therefore a suitable model to study branching, and perform lineage tracing [11] and genetic studies, as specific genes can be silenced by in vivo morpholino oligonucleotides or RNAi approaches, or overexpressed by viral transduction. The effects of these individual manipulations can be studied with in real-time and time-lapse mode, providing spatial-temporal characterization of nephrogenic induction and branching morphogenesis [12]. This system permits the screening of the CAKUT candidate genes in murine kidney explants at medium- to high-throughput efficiency.

Zebrafish are a promising model for studying kidney development in health and disease [13]. Zebrafish have key advantages that make it suitable for genetic research, including the generation of large numbers of offspring (ex utero) with rapid development, they are relatively inexpensive to maintain and easy to modify genetically. Additionally, modeling human genetic disease in zebrafish is greatly facilitated with recent advantages in genome editing techniques, including TALENs and CRISPR/Cas9. For kidney research specifically, zebrafish are suitable as zebrafish larvae have a pronephros 2-3 days post-fertilization (fig. 1b), which constitutes a simplified reflection of the human nephron [13]. The larval pronephros will be succeeded by a branched mesonephros, which in fish remains as the adult renal tissue. Although zebrafish do not develop a metanephros, the function of the genes that are involved in the development of the zebrafish pronephros is conserved in the mammalian metanephros [14]. Therefore, genetic factors found involved in zebrafish nephrogenesis will hold promise as human CAKUT gene candidates.

\section{Personalized Medicine and Stem Cell Lines}

Patient-specific disease modelling, drug treatment, and cell replacement therapy making use of patient-derived stem cells are major advances that can lead to tailored medical decisions and treatments for the individual patient. Stem cells have an unlimited replicative capacity and dif- 


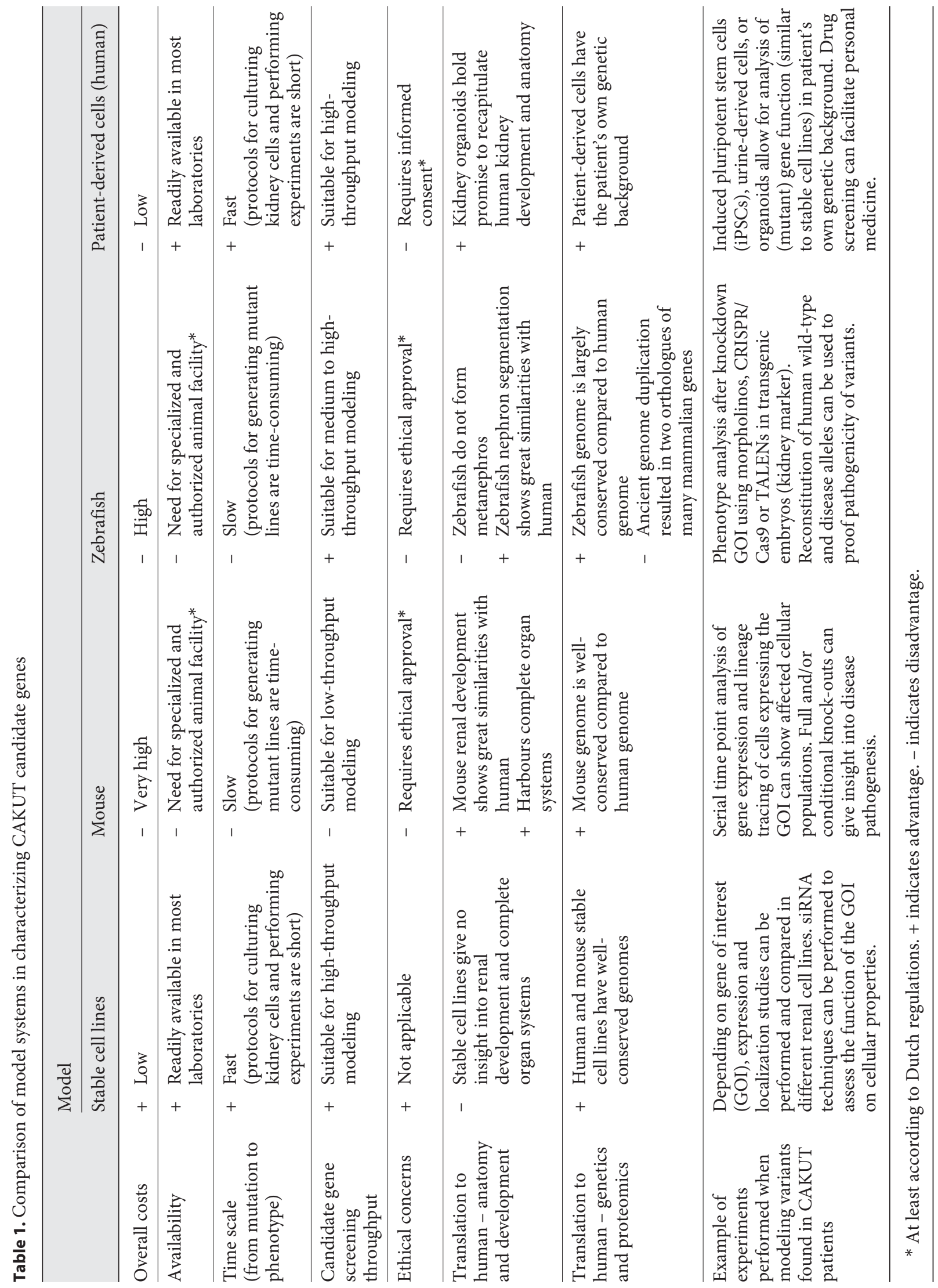




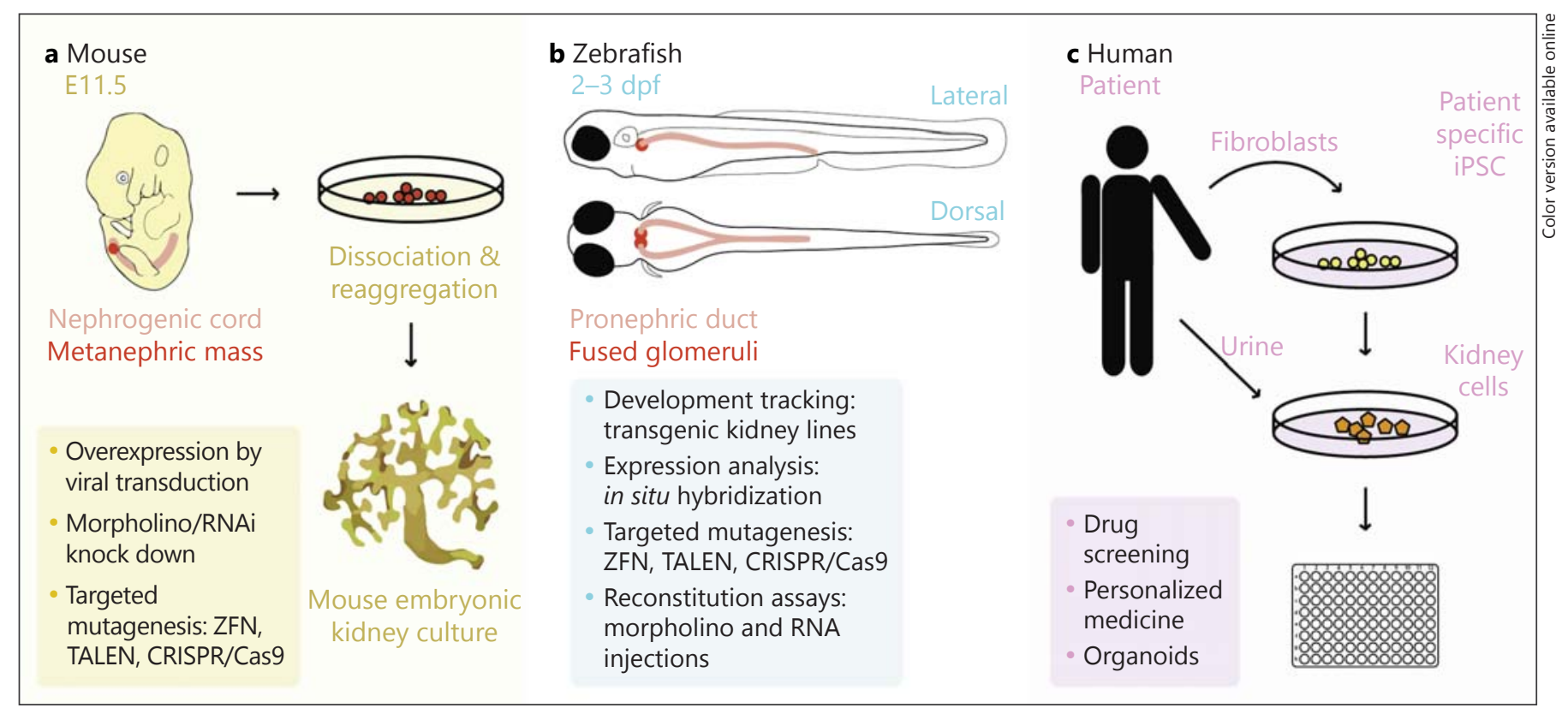

Fig. 1. Functional models for CAKUT. Schematic overview of different model systems for the functional characterization of novel CAKUT candidate genes. a In mice, mouse embryonic kidney cultures can be obtained by culturing cells of the metanephric mass (containing the metanephric mesenchyme; red) located at the posterior end of the nephrogenic cord (pink). Subsequently, these kidney cultures can be exposed to overexpression or knock-down of the CAKUT candidate genes and gene variants of interest. b In zebrafish, the embryonic kidney consists of two nephrons (pronephric ducts; pink) with the fused anterior glomeruli (red). Both nephrons fuse at the cloaca. The zebrafish embryo can be used for developmental tracking, expression analysis, targeted mutagenesis, and reconstitution assays of human alleles. c In human, novel genes involved in normal renal development might contribute to reprogramming of induced pluripotent stem cells (iPSC) into differentiated kidney cells. This methodology would enhance personalized medicine as renal cell lines (from differentiated iPSCs or urine-derived) and/or organoids could be cultured from individual patients and used for drug screening and fundamental research. ZFN; zinc finger nucleases, TALEN; transcription activator-like effector nucleases, CRISPR; clustered regularly interspaced short palindromic repeats. ferentiate into multiple cell types. Embryonic stem cells (ESCs) derive from the inner cell mass of fertilized eggs in mammals, whereas induced pluripotent stem cells (iPSCs) are adult somatic cells that have been reprogrammed to acquire a pluripotent state and subsequently differentiate into various cell types [15]. Stem cells facilitate the study of normal developmental processes and disease models that recapitulate human kidney disorders in a personal manner.

The generation of iPSC-derived kidney tissue from patient material, such as fibroblasts or urine-derived renal epithelial cells, leaves the genotype and phenotype intact, thereby enabling the development of a disease model in vitro that recapitulates human kidney disorders in a personalized manner $[16,17]$. Furthermore, iPSCs are a valuable tool for discovering and screening new drug compounds as well as testing patient-specific toxicity and adverse effects, thereby contributing to the development of personalized drug treatment (fig. 1c) [18]. Until recently, approaches to differentiate iPSCs in vitro led to low yields of kidney cell types, but more recent publications describe robust approaches for differentiating human iPSCs to ureteric bud kidney progenitor-like cells [17] and human ESCs into the ureteric bud and metanephric mesenchyme in vitro [19]. Interestingly, a simple, cost-effective and universal method for generating iPSCs was developed, using epithelial cells from urine [16]. This method represents a personalized and non-invasive tool for modelling genetic kidney diseases. Finally, gene editing has been successfully performed in these iPSCs as well [20].

Three-dimensional (3D) culture systems facilitate the differentiation of stem cells into structures resembling whole organs, known as organoids. They exhibit multiple cell types that self-assemble by cell sorting out, which is the reorganization of cell aggregates into homogeneous tissue domains, and spatially restricted lineage commitment of precursor cells [21]. The kidney tissue is capable of selforganization as well, driven by the growth of epithelial renal progenitor cells away from 2D culture and develop tu- 
bules and ducts when embedded in extracellular matrix hydrogels [22]. Cells derived from ureteric bud and metanephric mesenchyme are capable of growing in 3D culture [19]. Established organoids include the gut, kidney, brain, and retina, and provide a unique opportunity to study organ development and facilitate personalized disease modelling, tissue replacement therapy, and drug testing [21]. Organoids share some of the same advantages as patient-derived iPSC lines, such as the same genetic and phenotypic background of the patient, and are also amenable to all the standard experimental manipulations and analytical approaches. These include transfection, infection with recombinant retro- and lentiviruses, immunohistochemistry, gene expression analysis, mass spectrometry, genomic editing, live imaging, deep sequencing, and functional assays [23]. Renal organoids were successfully used to develop a polycystic kidney disease model through reprogramming fibroblasts from a patient toward iPSCs and subsequent differentiation that led to the aggregation and assembly of 3D chimaeric structures [17]. Organoids also have the potential to be used for the discovery of effective treatments or drugs, thereby, eliminating animal studies [21]. One example of successful replacement therapy comes from a study in which metanephric nephron progenitors were initially induced from mouse ESCs and reconstituted with 3D nephric tubules and glomeruli in culture.

Subsequently, cells were transplanted beneath the kidney capsule of immunodeficient mice, which resulted in tubulogenesis, the formation of glomeruli, and the integration of blood vessels into the transplanted tissue [24].

\section{Future Prospects for Human CAKUT Modeling}

For the Benefit of CAKUT Patients: High-Throughput and Personalized Models

Innovative research has led to the availability of in vivo, ex vivo, in vitro, and in silico model systems that are being applied in CAKUT research. Although we clearly benefit from these model systems, high-throughput systems are needed to characterize novel genes and variants, in order to unravel the underlying molecular mechanisms and increase the rate of clinical diagnosis in daily patient care. Ideally, the development of suitable models would lead to more accurate, more reliable, costeffective, and early diagnosis of CAKUT with good prospects on improved genetic counseling, clinical decisionmaking, and health outcomes at the individual level. Large-scale culturing and characterization of patientderived cells is very promising in this perspective. Mod- eling of the different phenotypic forms of CAKUT and the complex inheritance patterns involving multiple mutations in individual patients remains challenging. We believe that joint efforts in designing the appropriate models will eventually translate to the optimization of CAKUT classification and diagnostics.

\section{Need for Joint Efforts}

While numerous causal genes still await discovery, low incidence and fragmentation of data limit our knowledge on the etiology of complex diseases like CAKUT. Collaborative initiatives including multidisciplinary consortia that show major efforts in biobanking of large patient cohorts are essential to make this happen. Data sharing tools is an important field in development, moderating collaborations on large data sets. Consistent and deepphenotyping is crucial when it comes to determining genotype-phenotype correlations and for comparing patients' genetic data. We support opportunities for global implementation of an unambiguous phenotyping methodology such as The Human Phenotype Ontology (HPO) system [25]. HPO is powerful for defining phenotypic similarities in large study cohorts, which can be of great benefit to database searches and matchmaking efforts in order to find additional and similar patients [25].

\section{Conclusions}

The available model systems are not yet sufficient to characterize the huge amount of genetic variants identified in CAKUT patients. We stress the urgency for novel and innovative functional models and we see opportunities for models based on patient-derived cells in order to improve the diagnostic and therapeutic management of renal malformations, thereby stimulating personalized medicine in the nephrogenetics field.

\section{Acknowledgments}

The authors' research was supported by the European Community's Seventh Framework Programme FP7/2009 under grant agreements 305608 (EURenOmics) and 241955 (SYSCILIA) and the Consortium Programme of the Dutch Kidney Foundation under grant agreement CP11.18 (KOUNCIL).

\section{Disclosure Statement}

The authors have no conflicts of interest. van de Hoek/Nicolaou/Giles/Knoers/ Renkema/Bongers 


\section{References}

1 Schedl A: Renal abnormalities and their developmental origin. Nat Rev Genet 2007;8: 791-802.

-2 Costantini F, Kopan R: Patterning a complex organ: branching morphogenesis and nephron segmentation in kidney development. Dev Cell 2010;18:698-712.

$>3$ Vainio S, Lin Y: Coordinating early kidney development: lessons from gene targeting. Nat Rev Genet 2002;3:533-543.

4 Renkema KY, Stokman MF, Giles RH, Knoers $\mathrm{NV}$ : Next-generation sequencing for research and diagnostics in kidney disease. Nat Rev Nephrol 2014;10:433-444.

$\checkmark 5$ Davis TK, Hoshi M, Jain S: To bud or not to bud: the RET perspective in CAKUT. Pediatr Nephrol 2014;29:597-608.

6 Kaku Y, Ohmori T, Kudo K, Fujimura S, Suzuki K, Evans SM, Kawakami Y, Nishinakamura R: Islet1 deletion causes kidney agenesis and hydroureter resembling CAKUT. J Am Soc Nephrol 2013;24:1242-1249.

$>7$ Humbert C, Silbermann F, Morar B, Parisot M, Zarhrate M, Masson C, Tores F, Blanchet P, Perez MJ, Petrov Y, Khau Van Kien P, Roume J, Leroy B, Gribouval O, Kalaydjieva L, Heidet L, Salomon R, Antignac C, Benmerah A, Saunier S, Jeanpierre C: Integrin alpha 8 recessive mutations are responsible for bilateral renal agenesis in humans. Am J Hum Genet 2014;94:288-294.

-8 Muller U, Wang D, Denda S, Meneses JJ, Pedersen RA, Reichardt LF: Integrin alpha8beta1 is critically important for epithelial-mesenchymal interactions during kidney morphogenesis. Cell 1997;88:603-613.

$>9$ Aida T, Imahashi R, Tanaka K: Translating human genetics into mouse: the impact of ultra-rapid in vivo genome editing. Dev Growth Differ 2014;56:34-45.
10 Giuliani S, Perin L, Sedrakyan S, Kokorowski P, Jin D, De Filippo R: Ex vivo whole embryonic kidney culture: a novel method for research in development, regeneration and transplantation. J Urol 2008;179:365-370.

11 Short KM, Hodson MJ, Smyth IM: Tomographic quantification of branching morphogenesis and renal development. Kidney Int 2010;77:1132-1139.

12 Shan J, Jokela T, Skovorodkin I, Vainio S: Mapping of the fate of cell lineages generated from cells that express the Wnt4 gene by timelapse during kidney development. Differentiation 2010;79:57-64.

13 Drummond IA, Davidson AJ: Zebrafish kidney development. Methods Cell Biol 2010; 100:233-260.

14 Fukuyo Y, Nakamura T, Bubenshchikova E, Powell R, Tsuji T, Janknecht R, Obara T: Nephrin and Podocin functions are highly conserved between the zebrafish pronephros and mammalian metanephros. Mol Med Rep 2014;9:457-465.

15 Osafune K: In vitro regeneration of kidney from pluripotent stem cells. Exp Cell Res 2010;316:2571-2577.

16 Zhou T, Benda C, Dunzinger S, Huang Y, Ho JC, Yang J, Wang Y, Zhang Y, Zhuang Q, Li Y, Bao X, Tse HF, Grillari J, Grillari-Voglauer R, Pei D, Esteban MA: Generation of human induced pluripotent stem cells from urine samples. Nat Protoc 2012;7:2080-2089.

17 Xia Y, Nivet E, Sancho-Martinez I, Gallegos T, Suzuki K, Okamura D, Wu MZ, Dubova I, Esteban CR, Montserrat N, Campistol JM, Iz pisua Belmonte JC: Directed differentiation of human pluripotent cells to ureteric bud kidney progenitor-like cells. Nat Cell Biol 2013;15:1507-1515.
18 O’Neill AC, Ricardo SD: Human kidney cell reprogramming: applications for disease modeling and personalized medicine. J Am Soc Nephrol 2013;24:1347-1356.

19 Takasato M, Er PX, Becroft M, Vanslambrouck JM, Stanley EG, Elefanty AG, Little $\mathrm{MH}$ : Directing human embryonic stem cell differentiation towards a renal lineage generates a self-organizing kidney. Nat Cell Biol 2014;16:118-126.

20 Hou Z, Zhang Y, Propson NE, Howden SE, Chu LF, Sontheimer EJ, Thomson JA: Efficient genome engineering in human pluripotent stem cells using Cas9 from Neisseria meningitidis. Proc Natl Acad Sci U S A 2013. 110:15644-15649.

21 Lancaster MA, Knoblich JA: Organogenesis in a dish: modeling development and disease using organoid technologies. Science 2014; 345:1247125.

22 Montesano R, Schaller G, Orci L: Induction of epithelial tubular morphogenesis in vitro by fibroblast-derived soluble factors. Cell 1991; 66:697-711.

23 Sato T, Clevers H: Growing self-organizing mini-guts from a single intestinal stem cell: mechanism and applications. Science 2013; 340:1190-1194.

24 Taguchi A, Kaku Y, Ohmori T, Sharmin S, Ogawa M, Sasaki H, Nishinakamura R: Redefining the in vivo origin of metanephric nephron progenitors enables generation of complex kidney structures from pluripotent stem cells. Cell Stem Cell 2014;14:53-67.

25 Robinson PN, Kohler S, Bauer S, Seelow D, Horn D, Mundlos S: The Human Phenotype Ontology: a tool for annotating and analyzing human hereditary disease. Am J Hum Genet 2008;83:610-615. 